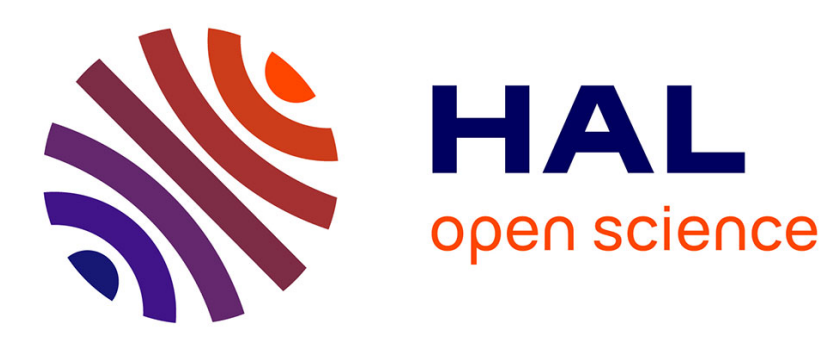

\title{
Equation-based interpolation and incremental unknowns for solving the three-dimensional Helmholtz equation
}

Pascal Poullet, Amir Boag

\section{To cite this version:}

Pascal Poullet, Amir Boag. Equation-based interpolation and incremental unknowns for solving the three-dimensional Helmholtz equation. 2012. hal-00763930

\section{HAL Id: hal-00763930 \\ https://hal.univ-antilles.fr/hal-00763930}

Preprint submitted on 11 Dec 2012

HAL is a multi-disciplinary open access archive for the deposit and dissemination of scientific research documents, whether they are published or not. The documents may come from teaching and research institutions in France or abroad, or from public or private research centers.
L'archive ouverte pluridisciplinaire HAL, est destinée au dépôt et à la diffusion de documents scientifiques de niveau recherche, publiés ou non, émanant des établissements d'enseignement et de recherche français ou étrangers, des laboratoires publics ou privés. 


\title{
Equation-based interpolation and incremental unknowns for solving the three-dimensional Helmholtz equation
}

\author{
Pascal Poullet ${ }^{1 *}$, Amir Boag $^{2}$ \\ ${ }^{1}$ LAboratoire de Mathématiques Informatique et Applications (LAMIA), Université des Antilles et de la Guyane, \\ BP 250, F-97157 Pointe-à-Pitre Cédex, Guadeloupe FWI \\ ${ }^{2}$ School of Electrical Engineering, Tel Aviv University, Tel Aviv 69978, Israel
}

\section{SUMMARY}

In an earlier paper [1], we developed an efficient incremental unknowns (IU) preconditioner for solving the two-dimensional (2D) Helmholtz problem in both high and low frequency (wavenumber) regimes. The multilevel preconditioning scheme involves separation of each grid into a coarser grid of the following level and a complementary grid on which the IUs are defined by interpolation. This approach is efficient as long as the mesh size of the coarsest grid is sufficiently small compared to the wavelength. In order to overcome this restriction, the authors introduced recently (in [2]) a modified IU method combining the conventional interpolation with the Helmholtz equation based interpolation (EBI). The EBI coefficients are derived numerically using a sufficiently large set of analytic solutions of the Helmholtz equation on a special hierarchy of stencils. The modified IUs using Helmholtz EBI are shown to provide improved preconditioning on the coarse scales where the conventional interpolation can not be employed. This study deals with the extension of this idea for solving the three-dimensional (3D) Helmholtz equation. Copyright (C) 0000 John Wiley \& Sons, Ltd.

Received ...

KEY WORDS: Helmholtz equation; Iterative methods; Preconditioning; Multilevel methods; Incremental Unknowns

\section{INTRODUCTION}

Various applications such as oil exploration, analysis of optical devices, as well as marine and aero acoustics require efficient methods to compute numerical solutions of the Helmholtz equation. This equation,

$$
\Delta E+k^{2} E=f
$$

equipped with various boundary conditions, is obtained by applying the Fourier transform with respect to time to the acoustic wave equation. In (1), $E$ represents the unknown field, $f$ is the volumetric source term, and $k$ denotes the wavenumber related to the temporal frequency $(\omega)$ by $k=\omega / c$ where $c$ is the wave propagation velocity. When equation (1) is satisfied with a zero right hand side (RHS), the excitation of the problem is provided by the boundary conditions. This equation appears in many geometric configurations, but we will focus on solving it in a threedimensional unbounded domain. Hence, to avoid reflections, absorbing boundary conditions (ABC)

*Correspondence to: LAboratoire de Mathématiques Informatique et Applications, Université des Antilles et de la Guyane, BP 250, F-97157 Pointe-à-Pitre Cédex, Guadeloupe FWI. E-mail: Pascal.Poullet@ univ-ag.fr

Contract/grant sponsor: This research was supported in part by a grant from the Ministry of Science, Culture \& Sport, Israel \& the Ministry of Research, France.

Copyright (c) 0000 John Wiley \& Sons, Ltd.

Prepared using nlaauth.cls [Version: 2010/05/13 v2.00] 
will be provided on the outer boundary of the solution domain. As the $\mathrm{ABC}$ are necessarily complex, a finite difference approximation of the Helmholtz boundary value problem typically leads to a large-scale indefinite complex linear system:

$$
A e=b \quad \text { with } A \in \mathbb{C}^{n \times n}, \quad \text { and } \quad e, b \in \mathbb{C}^{n}
$$

where $e$ denotes the unknown discrete field, while the known RHS vector $b$ stands for the excitation. The latter can be provided by the boundary conditions on the surface of a scatterer, the $\mathrm{ABC}$, or the RHS of equation (1) if the source term is explicitly included. To obtain a meaningful solution, the spatial mesh size $h$ must be sufficiently small; usually one takes $k h<\pi / 5$, which corresponds to $h$ of less than one tenth of the wavelength ([3]). Consequently, challenging problems occur when the wavenumber is high. For 2D configurations, the computation of numerical solutions of such a problem can be performed by using either direct or iterative solvers. However, in 3D configurations, the choice of direct methods such as the LU decomposition is often no longer feasible due to the large size of the pertinent matrices.

Though a variety of iterative methods for solving the Helmholtz equation have been introduced over the years (see [4] for a review of most common techniques), this research area remains very active. In particular, a class of $2 \mathrm{D}$ iterative solvers using a Helmholtz-type differential operator as a preconditioner has been recently introduced in $[5,6,7]$, which appears to produce the major advance in this field. However, to solve three-dimensional problems, it is often necessary to process very large linear systems. In such situations even building and storing an incomplete factorization might become a computational bottleneck. Moreover, to solve an indefinite problem such as the Helmholtz one with multigrid techniques can be a hard task $[8,9,10]$. Indeed, depending on the wavenumber, the coarsening must be limited to catch the small eigenfrequencies of the solution, and a suitable choice of the smoothing operator is needed to obtain a quick convergence $[11,8,6]$.

During the late 80's, the Incremental Unknowns method was developed, for the dynamic system analysis, with the aim of modeling long-term dynamic behavior of dissipative evolutionary equations [12]. By introducing a simple multilevel basis creating a hierarchy of unknowns for the finite difference schemes (closely linked to the hierarchical bases in the finite element context [13] or wavelet bases), this multilevel method facilitates an application of iterative solvers such as the conjugate gradient algorithm to the systems in this new basis. A number of studies have been conducted to analyze and prove the efficiency of this tool as a preconditioner for solving elliptic partial differential equations $([14,15,16]$ in $2 \mathrm{D}$, and $[17,18,19]$ in $3 \mathrm{D})$, similar to classical multigrid methods. Other works have demonstrated that it is possible to modify this tool to efficiently solve other linear problems [20, 21, 22, 1]. In particular, it has been shown that such multilevel schemes can be efficient for solving an exterior Helmholtz problem [1]. Results obtained by the authors were in agreement with the previous studies of indefinite elliptic boundary value problems $[23,24]$. It has been proven that for the best efficiency, the number of IU levels should be designed for the coarsest grid to have roughly two points per linear wavelength [1]. In order to improve on this first result, the authors developed a new method by updating the coarsening strategy at the transition level beyond which the coarse grids can not meaningfully represent the solution [2]. To define this new coarsening strategy a hierarchy of square cells (boxes) is considered on the grid points of the transition level. New incremental unknowns using Helmholtz equationbased interpolation (EBI) are introduced at all levels starting with the transition level. These EBIIU are computed on a cross of grid points at the middle of each box and they use interpolation from the points located on the boundary of the cell. As this new strategy demonstrated significant improvements in preconditioning for one and two-dimensional Helmholtz problem, this paper deals with the extension and analysis of the method for three-dimensional domains. In this context, the computational domain is split into a hierarchy of cubic cells. The EBI-IU class is then composed of three orthogonal planes as a natural extension of the cross in 2D, while the class of the cubic box boundaries appears as an analog of the square contours in 2D [2].

The paper is organized as follows. First, the multilevel decomposition that we use for the 3D Helmholtz equation is introduced in the following section. Here, we describe a generic transition between $3 \mathrm{D}$ fine and coarse grids with the conventional IUs being defined on the complementary 
grid. Subsequently, we introduce the EBI-IU scheme for a multilevel hierarchy of cubic cells. Section 3 is devoted to presenting our model problem, the scheme used to compute its approximate solution and the numerical results. Some concluding remarks are presented in the last section.

\section{MULTILEVEL DECOMPOSITION}

\subsection{The Incremental Unknowns in $3 D$}

We first present the Incremental Unknowns (IUs) formulation for the case of two grids in three dimensions. Let $G_{h}$ denote the original fine grid comprising $(m+1) \times(m+1) \times(m+1)$ points with with mesh size $h$ and $G_{2 h}$ refer to a grid with mesh size $2 h$ whose $(m / 2+1) \times(m / 2+1) \times$ $(m / 2+1)$ points coincide with the even points of $G_{h}$. We assume that $m$ is a multiple of 2 and the grid point indices range from 0 to $m$ and from 0 to $m / 2$ for $G_{h}$ and $G_{2 h}$, respectively. The IU structure is as follows: the coarse grid $G_{2 h}$ consists of the $G_{h}$ nodes of type $(2 i, 2 j, 2 l)$, where $i, j, l=0, \cdots, m / 2$ and the complementary grid $G_{f}=G_{h} \backslash G_{2 h}$ whose nodes serve to define the IUs. This complementary grid comprises seven different families of nodes: the nodes located at mid edge of the coarse grid in each of the three directions, the nodes located at a mid face of the closest neighbours of the coarse grid in the three planes and the nodes at the middle of two planes defined by the height closest neighbours of the coarse grid. Specifically:

(a) the $(2 i, 2 j, 2 l+1)$ nodes are located at mid edge transversally between two nodes of the coarse grid (type (a), in FIG. 1);

(b) the $(2 i+1,2 j, 2 l)$ nodes are located at mid edge laterally between two nodes of $G_{2 h}$ (type (b) in the same figure);

(c) the $(2 i, 2 j+1,2 l)$ nodes are located at mid edge vertically between two nodes of $G_{2 h}$ (type (c) in the same figure);

(d) the $(2 i+1,2 j, 2 l+1)$ nodes are located at mid face laterally at the middle among four nodes of $G_{2 h}$ (type (d) in the same figure);

(e) the $(2 i, 2 j+1,2 l+1)$ nodes are located at mid face transversally at the middle among four nodes of $G_{2 h}$ (type (e) in the same figure);

(f) the $(2 i+1,2 j+1,2 l)$ nodes are located at mid face vertically at the middle among four nodes of $G_{2 h}$ (type (f) in the same figure);

(g) the $(2 i+1,2 j+1,2 l+1)$ nodes are located at the middle of two planes defined by the height neighbours (type ( $\mathrm{g}$ ) in the same figure).
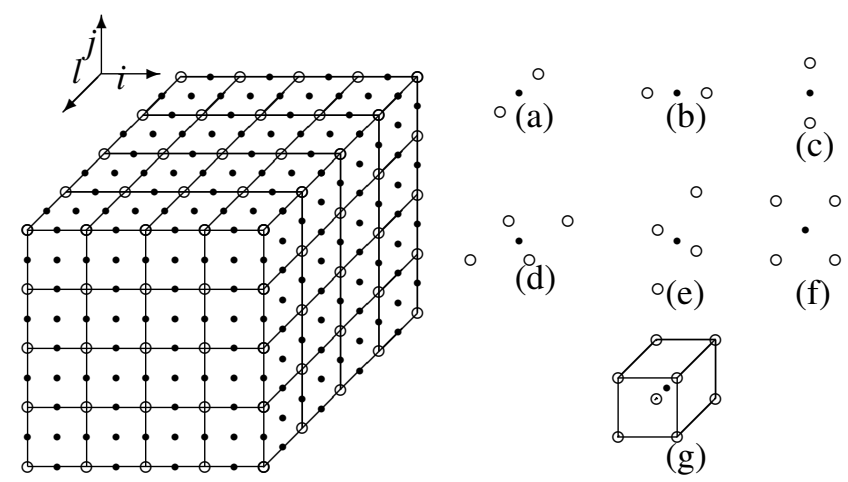

Figure 1. An example of a mesh split in two grids, for $m=8$. The coarse grid points (o) are gathered in $G_{2 h}$, the points $(\bullet)$ are gathered in the complementary grid $G_{f}$. This complementary grid is composed of seven families of nodes: type (a) are $(2 i, 2 j, 2 l+1)$ nodes, type (b) - $(2 i+1,2 j, 2 l)$ nodes, type (c) $-(2 i, 2 j+1,2 l)$ nodes, type (d) - $(2 i+1,2 j, 2 l+1)$ nodes, type (e) $-(2 i, 2 j+1,2 l+1)$ nodes, type (f) $-(2 i+1,2 j+1,2 l)$ nodes, and type $(\mathrm{g})-(2 i+1,2 j+1,2 l+1)$ nodes. 
If $e$ designates the unknown field of the initial system, let $y$ be the unknown of the coarse grid and $z$ - the small correction relative to the interpolated values defined by the IU on the complementary grid $\left(G_{f}\right)$. Following Chen and Temam [17], we define $y$ and $z$ as:

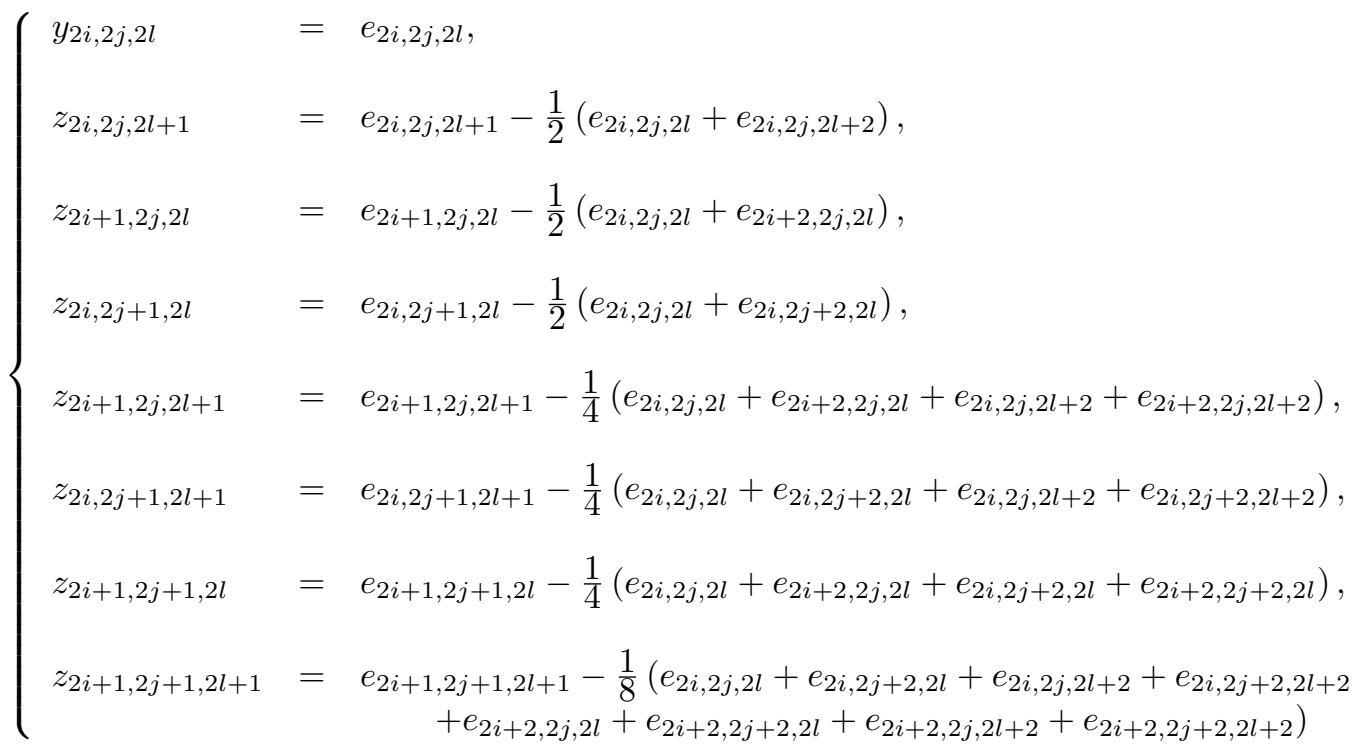

Gathering in a vector $\bar{e}^{1}$ the new unknowns: $\quad y_{2 i, 2 j, 2 l}, z_{2 i, 2 j, 2 l+1}, z_{2 i+1,2 j, 2 l}, z_{2 i, 2 j+1,2 l}$, $z_{2 i+1,2 j, 2 l+1}, z_{2 i, 2 j+1,2 l+1}, z_{2 i+1,2 j+1,2 l}, z_{2 i+1,2 j+1,2 l+1}$, the formulas introduced previously can be inverted and expressed as a linear transformation:

$$
e=S_{1} \bar{e}^{1}
$$

which defines matrix $S_{1}$. The structure of this matrix is sparse and very simple because, subject to reordering the unknowns, it can be written as $I$ (the identity matrix of $\mathcal{M}_{m+1^{3}}(\mathbb{R})$ ) plus the negative of the interpolation coefficients of (3). After reordering the unknowns in the lexicographical order, at each line corresponding to a coarse grid unknown, only the diagonal value is not zero and equals to one. Whereas, at each line corresponding to an unknown of grid $G_{f}$, for example, of the $(2 i, 2 j, 2 l+1)$ type, we have, in additional to the one on the main diagonal, two other nonzero values (on both sides of the diagonal), each equal to $1 / 2$ at the two columns which represent the unknowns of the $G_{2 h}$ neighbors.

To extend this construction to a multilevel structure, we consider nested grids by dyadic decomposition. After $d$ levels of IU grid coarsening, one obtains the coarsest grid with $\left(m_{d}+1\right) \times$ $\left(m_{d}+1\right) \times\left(m_{d}+1\right)$ points where $m_{d}=m / 2^{d}$ and mesh size equals $h_{d}=l / m_{d}=2^{d} h$. Let the unknowns on the original grid be referred to as $y^{0}=\bar{e}^{0}=e$. Then, for transition between level $d$ and level $d-1$, by analogy with formula (4), we get for $d \geq 1$ : 


$$
\begin{aligned}
y_{2 i, 2 j, 2 l+1}^{d-1} & =z_{2 i, 2 j, 2 l+1}^{d}+\frac{1}{2}\left(y_{2 i, 2 j, 2 l}^{d}+y_{2 i, 2 j, 2 l+2}^{d}\right) \\
y_{2 i+1,2 j, 2 l}^{d-1} & =z_{2 i+1,2 j, 2 l}^{d}+\frac{1}{2}\left(y_{2 i, 2 j, 2 l}^{d}+y_{2 i+2,2 j, 2 l}^{d}\right) \\
y_{2 i, 2 j+1,2 l}^{d-1}= & z_{2 i, 2 j+1,2 l}^{d}+\frac{1}{2}\left(y_{2 i, 2 j, 2 l}^{d}+y_{2 i, 2 j+2,2 l}^{d}\right), \\
y_{2 i+1,2 j, 2 l+1}^{d-1}= & z_{2 i+1,2 j, 2 l+1}^{d}+\frac{1}{4}\left(y_{2 i, 2 j, 2 l}^{d}+y_{2 i+2,2 j, 2 l}^{d}+y_{2 i, 2 j, 2 l+2}^{d}+y_{2 i+2,2 j, 2 l+2}^{d}\right), \\
y_{2 i, 2 j+1,2 l+1}^{d-1}= & z_{2 i, 2 j+1,2 l+1}^{d}+\frac{1}{4}\left(y_{2 i, 2 j, 2 l}^{d}+y_{2 i, 2 j+2,2 l}^{d}+y_{2 i, 2 j, 2 l+2}^{d}+y_{2 i, 2 j+2,2 l+2}^{d}\right) \\
y_{2 i+1,2 j+1,2 l}^{d-1}= & z_{2 i+1,2 j+1,2 l}^{d}+\frac{1}{4}\left(y_{2 i, 2 j, 2 l}^{d}+y_{2 i+2,2 j, 2 l}^{d}+y_{2 i, 2 j+2,2 l}^{d}+y_{2 i+2,2 j+2,2 l}^{d}\right) \\
y_{2 i+1,2 j+1,2 l+1}^{d-1}= & z_{2 i+1,2 j+1,2 l+1}^{d}+\frac{1}{8}\left(y_{2 i, 2 j, 2 l}^{d}+y_{2 i, 2 j+2,2 l}^{d}+y_{2 i, 2 j, 2 l+2}^{d}+y_{2 i, 2 j+2,2 l+2}^{d}\right. \\
& \left.+y_{2 i+2,2 j, 2 l}^{d}+y_{2 i+2,2 j+2,2 l}^{d}+y_{2 i+2,2 j, 2 l+2}^{d}+y_{2 i+2,2 j+2,2 l+2}^{d}\right) .
\end{aligned}
$$

Relations (5) can be expressed in a matrix form as

$$
y^{d-1}=\bar{S}_{d}\left[\begin{array}{l}
y^{d} \\
z^{d}
\end{array}\right] .
$$

Then, $\bar{e}^{d-1}=S_{d} \bar{e}^{d}$ where

$$
\bar{e}^{d}=\left[\begin{array}{c}
y^{d} \\
z^{d} \\
z^{d-1} \\
\vdots \\
z^{1}
\end{array}\right] \quad \text { and } \quad S_{d}=\left[\begin{array}{cc}
\bar{S}_{d} & 0 \\
0 & I .
\end{array}\right]
$$

Note that $\bar{e}^{d}$ comprises the unknowns $y^{d}, z^{d}$ of the $d^{t h}$ grid $G_{h_{d}}$ as well as $z^{l}, l=1, \cdots, d-$ 1 of the preceding levels. Applying the IU definitions (5)-(7) recursively, the whole $d$-level decomposition can be expressed as:

$$
e=S_{1} S_{2} \bar{e}^{2}=\underbrace{S_{1} S_{2} \ldots S_{d}}_{S} \bar{e}^{d} .
$$

Using equation (8), we are able to define multilevel preconditioning schemes with the IUs. The simplicity of this formula indicates that this process of basis transfer from the nodal basis to the IU basis can be performed directly by an iterative solver, requiring no additional storage.

Let $A$ be the matrix of the original linear problem and let $S$ be the transfer matrix defined in (8). The structure of the real-valued matrix $S$ is studied in detail in [19]. One can introduce matrix $S$ in two ways: either by considering the normal equation system writen in the incremental unknowns basis, or by setting the original system in the IU basis. In the first case, the system to solve is the following

$$
S^{T} A^{*} A S \bar{e}=S^{T} A^{*} b \quad \text { with } S \bar{e}=e
$$

where $\bar{e}$ is the vector of Incremental Unknowns. Note that we eliminated the superscript on $\bar{e}$ explicitly indicating the number of IU levels. To obtain a Hermitian system to solve, one derives (9) by left-multiplying the normal equations with $S^{T}$.

In the case of some elliptic problems (Poisson equation), it has been proved in [17] that if $C$ is the matrix of the discrete linear problem (with Dirichlet boundary conditions) and $\kappa(C)$ - its condition 
number, we have

$$
\kappa\left(S^{T} C S\right) \ll \kappa(C)
$$

Specifically, if $h$ denotes the mesh size on the fine grid, they proved that the condition number of the matrix $S^{T} C S$ has the asymptotic behavior $O\left(h^{-1}(\log h)^{4}\right)$, i.e., significantly better than that $C$, which grows as $O\left(h^{-2}\right)$. However, compared to the condition number estimates for the 2D problem [15], this result indicates that the improvement generated by the IU preconditioning in $3 \mathrm{D}$ is lower.

\subsection{Three-dimensional IUs using equation-based interpolation}

The IU described in the previous section are expected to be effective for preconditioning as long as the mesh size of the coarsest grid is sufficiently small compared to the wavelength. This conclusion stems from the fact that the IU are based on interpolation, while solutions to the Helmholtz equation can not be meaningfully interpolated on a uniform grid when the mesh size is equal or larger than half the wavelength as discussed in [1]. To that end, we develop a three dimensional (3D) equationbased interpolation (EBI) as an extension of our earlier work [2]. Let us recall that in the IU approach the multilevel nested structure at each level must be decomposed into two classes of nodes (linked to the solution values at these points): first, the reference class which includes the nodes from which the interpolation will be computed, and second, the incremental class that comprises the target nodes for which the interpolation will be computed. Note that the IUs are defined as a difference between the actual solution and the interpolated values at the nodes of the incremental class.

The EBI is constructed as a discrete approximation of an integral representation for a solution to the Helmholtz equation in a bounded sub-domain. Thus, for a given sub-domain, the boundary values sampled with a sufficient density can be used to determine the solution at the interior points. In 3D, we consider cubical (box) sub-domains filling the whole solution domain. One can then define a hierarchy of nested sub-domains by setting the nodes on the sub-domains' boundaries as the reference class of points, and the incremental class comprising nodes located on the three interior planes at the middle of each box (if $O$ is at the center of a box, the planes are $x O y, x O z$, $y O z$ ). Similarly to the approach in $2 \mathrm{D}$ for which the two classes are the border and the cross [2], the incremental class in 3D comprises nodes of the 3-cross which is the cross in 3D (cf. 2).

$t$ Like in [2], the EBI is designed specifically for the Helmholtz equation by using its solutions. In general, a solution of the Helmholtz equation in a cube can be expressed as

$$
E(x, y, z)=\int_{0}^{2 \pi} \int_{0}^{\pi} W(\theta, \phi) \Psi_{\theta, \phi}(x, y, z) d \theta d \phi,
$$

where

$$
\Psi_{\theta, \phi}(x, y, z)=\exp \{\imath k(x \sin \theta \cos \phi+y \sin \theta \sin \phi+z \cos \theta)\}
$$

is referred as a plane wave with $\theta$ and $\phi$ represents respectively the elevation and azimuth of the direction of propagation. For a specific solution $E(x, y, z)$, function $W(\theta, \phi)$ represents the weight of the $(\theta, \phi)$-directed plane wave in the spectral representation (11). Thus, if an interpolation rule were satisfied by $\Psi_{\theta, \phi}(x, y, z)$ for all $0 \leq \theta<\pi, 0 \leq \phi<2 \pi$, it would provide a perfect interpolation for any $E(x, y, z)$ satisfying the Helmholtz equation. In designing an interpolation scheme involving $J$ points $\left\{\left(x_{j}^{b}, y_{j}^{b}, z_{j}^{b}\right), j=1, \ldots, J\right\}$ of the cube boundary, it is sufficient to use a subset of $P \geq J$ plane waves $\left\{\Psi_{\theta_{p}, \phi_{p}}(x, y, z), p=1, \ldots, P\right\}$ with the propagation directions $\left(\theta_{p}, \phi_{p}\right)$ uniformly spaced over the unit sphere. We require that the interpolation rule is satisfied in the least squares sense for the $P$ plane waves (12) to obtain an over-determined system of equations for each target point. Specifically, for each point $\left\{\left(x_{i}^{c}, y_{i}^{c}, z_{i}^{c}\right), i=1, \ldots, s\right\}$ of the three internal median planes, we obtain a $P \times J$ system

$$
\forall 1 \leq p \leq P, \quad \sum_{j=1}^{J} \Psi_{\theta_{p}, \phi_{p}}\left(x_{j}^{b}, y_{j}^{b}, z_{j}^{b}\right) w_{j}^{i}=\Psi_{\theta_{p}, \phi_{p}}\left(x_{i}^{c}, y_{i}^{c}, z_{i}^{c}\right),
$$

to be solved in the least squares sense for the interpolation weights $w_{j}^{i}$. In fact, the numerical rank of the system is often lower than $J$ due to the proximity of $k$ to one of the eigenvalues of the 


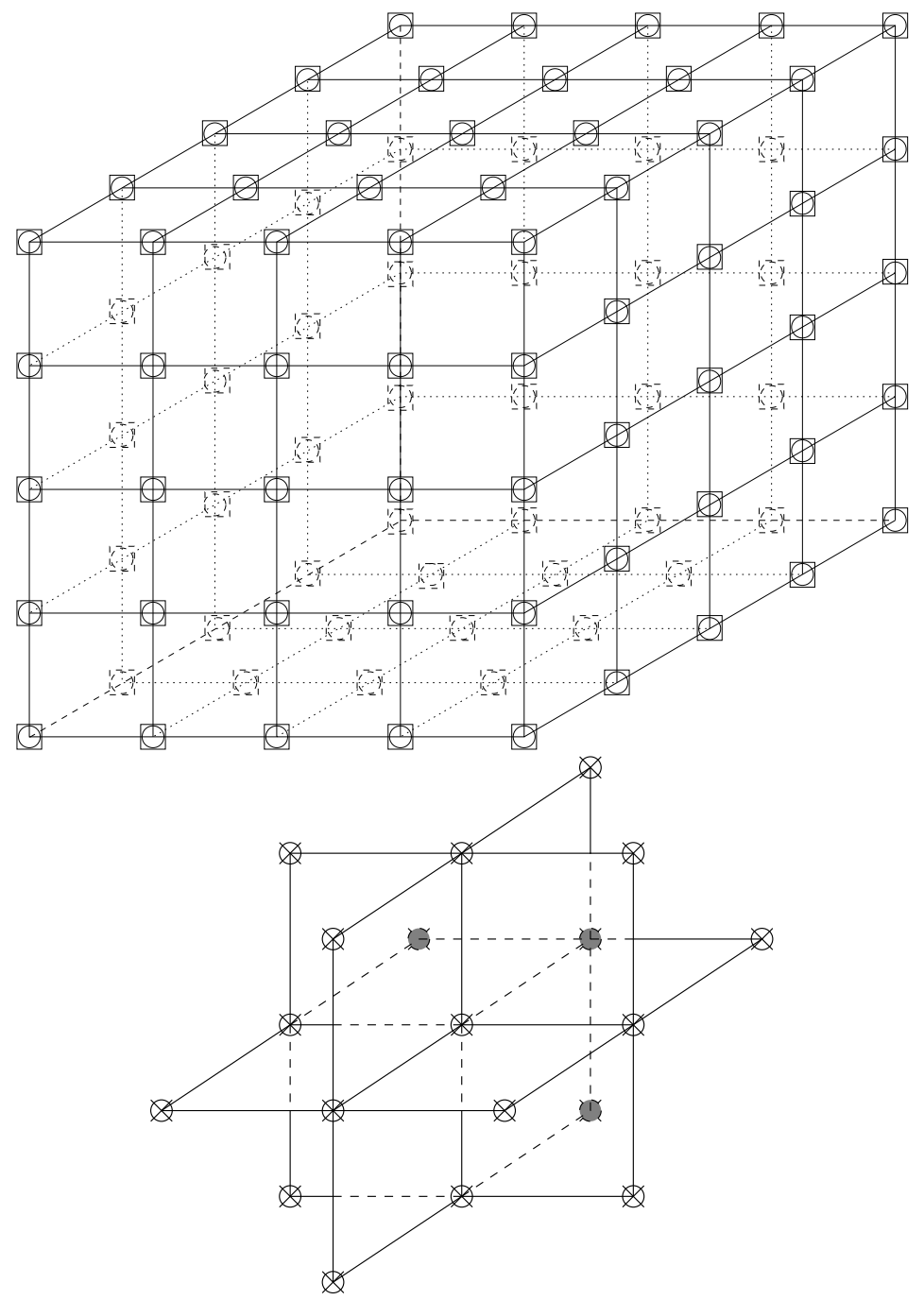

Figure 2. Illustration of the equation-based interpolation (EBI): (a) Reference class nodes from which the EBI values are computed form an array of cubic cells of the bullet nodes referred as $\mathcal{B}_{l}$; (b) Location of the incremental class nodes where the field is computed by the equation-based interpolation is defined as $\mathcal{C}_{l}=\mathcal{B}_{l-1} \backslash \mathcal{B}_{l}$ and forms an intersection of three planes inside each cubic cell.

Helmholtz problem with Dirichlet boundary conditions on a given square domain. To that end, we seek a regularized solution for system (13) via the singular value decomposition (SVD).

The EBI-IUs can now be defined by analogy to (3) as a difference between the actual and interpolated values:

$$
\begin{aligned}
& Y_{\{j\}}^{b}=E\left(x_{j}^{b}, y_{j}^{b}, z_{j}^{b}\right), \\
& Z_{\{i\}}^{c}=E\left(x_{i}^{c}, y_{i}^{c}, z_{i}^{c}\right)-\sum_{j=1}^{J} w_{j}^{i} E\left(x_{j}^{b}, y_{j}^{b}, z_{j}^{b}\right),
\end{aligned}
$$

with the subscript $\{j\}$ stands for the $j^{\text {th }}$ unknown value on the cube border among the $J$ values. The subscript $\{i\}$ stands for the $i^{\text {th }}$ value which is defined among the $s$ values of the 3D-cross (intersections of three planes).

The EBI-IUs construction is performed in a multilevel fashion, though here, for the sake of clarity, we illustrated the process using a simplified notation. 


\section{THE MULTILEVEL SOLVER}

In this section, the model problem is defined and its approximation by a finite difference scheme is given. Subsequently, numerical results illustrating the solution of our model problem are presented with the goal of studying and optimizing the performance of our multilevel method.

\subsection{A scattering test problem}

Let us denote by $\Pi=]-2,2\left[{ }^{3}\right.$, an open origin-centered cube, and by $\Omega$, the domain that is contained in $\Pi$ and obtained by removing from thereof a small concentric corpus. Then, if one considers that this corpus represents a perfectly soft acoustic scatterer located in an unbounded domain, our scattering test problem can be approximated as follows:

$$
\left\{\begin{array}{rlll}
\Delta E+k^{2} E & = & & \text { in } \Omega=\Pi \backslash \bar{\Sigma} \\
E & =f & & \text { on } \bar{\Sigma} \\
\mathcal{B} E & = & & \text { on } \partial \Pi
\end{array}\right.
$$

where the operator $\mathcal{B}$ corresponds to the chosen absorbing boundary condition $(\mathrm{ABC})$ that facilitates the replacement of an unbounded problem by the finite size computational domain $\Omega$. The second equation in (16) represents a perfectly soft acoustic scatterer with $f$ being the negative of the incident field. In agreement with the previous studies in 2D or 3D ([1], [2], [25]), when using a second order centered scheme for the Laplace operator, the accuracy of the solution is not affected by expressing the $\mathrm{ABC}$ by the second-order approximation due to Mur [26]. To that end, let the boundary of the cube $\partial \Pi$ be split into six square faces $\Gamma_{ \pm l}, l=1,2,3$ whose outward normal directions are given by the coordinate directions $\pm x_{l}$ ). The second-order $\mathrm{ABC}$ on the interior points of these faces $\left(\Gamma_{ \pm l}, l=1,2,3\right)$ reads

$$
\mathcal{B}_{i n t} E \equiv \pm \frac{\partial E}{\partial x_{l}}-\imath k E-\frac{\imath}{2 k} \sum_{1 \leq j \neq l \leq 3} \frac{\partial^{2} E}{\partial x_{j}^{2}}=0 .
$$

Furthermore, according to the study [27], the singular points of the boundary require a different treatment. In particular, the following condition is employed on the edge between the faces $\Gamma_{ \pm m}$ and $\Gamma_{ \pm j}$, denoted as $\Gamma_{( \pm m, \pm j)}$ :

$$
\mathcal{B}_{\text {edge }} E \equiv-\frac{3}{2} k^{2} E-\imath k\left( \pm \frac{\partial E}{\partial x_{m}} \pm \frac{\partial E}{\partial x_{j}}\right)-\frac{1}{2} \frac{\partial^{2} E}{\partial x_{l}^{2}}=0 .
$$

In addition, the $\mathrm{ABC}$ approximation

$$
\mathcal{B}_{\text {corn }} E \equiv-2 \imath k E+\sum_{j=1}^{3} \pm \frac{\partial E}{\partial x_{j}}=0
$$

should be satisfied at each of the eight corners of the cube $\bar{\Pi}$.

\subsection{Numerical results}

In this section, we compare the performance of different multilevel methods combining the IU and EBI-IU preconditioning. These methods are applied to solve the scattering boundary value problem (16). At the center of the domain, the small box which plays the role of a perfectly soft acoustic scatterer, is illuminated by a plane wave $e^{\imath k x}$, i.e., we have $f=-e^{\imath k x}$ over $\Sigma$. All methods use the conjugate gradient (CG) method for solving normal equations (referred as the CGNR algorithm on p. 236 in Ref. [28] and recently used in [2, 1,29]) with various multilevel preconditioners introduced in the previous sections.

Table I is devoted to presenting the influence of the wavelength/wavenumber on the performance of different methods based on the conventional IU preconditioning. To assign a name to a method, 
a digit is added to "CGIU" to represent the number of IU-levels used in the preconditioner. Then, with the total number of grid points being fixed, the best result is obtained usually by the method whose coarsest grid corresponds to the density of two points per wavelength. For example, consider in Table I the case of $\lambda=0.25$, which makes the length of the domain to comprise 16 wavelengths. We can see that the most efficient method in this case is CGIU3. Indeed, 256 grid points in each direction after 3 levels of IU reduce to 32 on the coarsest grid, thus reaching the threshold of 2 points per wavelength. This rule, however is not perfectly accurate, because for $\lambda=1.0$, the solver that uses 4 grid levels of IU gives slightly better results than CGIU5. Thus, we conclude that while the limit of 2 points per wavelength is definitely a lower bound, coarse grids with 4 points can sometimes be more appropriate. A similar phenomenon has been observed in $2 \mathrm{D}$, where we also demonstrated that if more IU levels, beyond the 2 point per wavelength threshold are added, the preconditioner performance tends to deteriorate $[1,2]$.

\begin{tabular}{|l||c|c|c|c|}
\hline & $\lambda=0.25$ & $\lambda=0.5$ & $\lambda=1.0$ & $\lambda=2.0$ \\
\hline CGIU1 & 2601 & 8160 & 11397 & $* * *$ \\
\hline CGIU2 & 1028 & 2930 & 4091 & 9770 \\
\hline CGIU3 & 774 & 1256 & 1687 & 3953 \\
\hline CGIU4 & 804 & 1020 & 1250 & 3264 \\
\hline CGIU5 & 1768 & 1074 & 1274 & 2962 \\
\hline CGIU6 & 3465 & 1170 & 1334 & 3768 \\
\hline CGIU7 & 5023 & 1670 & 1399 & 4041 \\
\hline CGIU8 & 5490 & 1865 & 1404 & 4051 \\
\hline
\end{tabular}

Table I. Number of iterations required for the iterative methods to achieve convergence (i.e., to reduce the relative $L_{2}$ norm of the residual $\|b-A x\|_{2} /\|b\|_{2}$ below $5.10^{-7}$ ) for various values of the wavelength $(\lambda=2 \pi / k)$. Case where convergence has not been achieved within 25,000 iterations is marked with $* * *$. The computations are performed on a grid comprising $257^{3}$ points.

Similarly, Table II gathers the result of the same test problem with the conventional IU preconditioning that has been solved previously ( $c f$. Table I), but for the finest grid that is two times coarser. Regardless of the value of the wavelength, the results that we obtained, proved that the most efficient method reaches the density of two points per wavelength for the coarsest grid.

\begin{tabular}{|l||c|c|c|}
\hline & $\lambda=0.25$ & $\lambda=0.5$ & $\lambda=1.0$ \\
\hline CG & 1907 & 3153 & 6703 \\
\hline CGIU1 & 695 & 1100 & 2326 \\
\hline CGIU2 & 515 & 472 & 868 \\
\hline CGIU3 & 696 & 396 & 475 \\
\hline CGIU4 & 1737 & 412 & 472 \\
\hline CGIU5 & 3308 & 708 & 509 \\
\hline CGIU6 & 4833 & 1043 & 529 \\
\hline
\end{tabular}

Table II. Number of iterations required for the iterative methods to achieve convergence (i.e., to reduce the relative $L_{2}$ norm of the residual $\|b-A x\|_{2} /\|b\|_{2}$ below $5.10^{-7}$ ) for various values of the wavelength $(\lambda=2 \pi / k)$. The computations are performed on a grid comprising $129^{3}$ points.

In Table III, we summarize the results obtained with various methods which combine the conventional IU with the multilevel EBI, applied to the problem (16) with $k=2 \pi$. The results show that for this value of the wavenumber, the most efficient method is obtained by adding multilevel EBI-IU to the conventional 4-level IU preconditioner, which yields the coarsest grid density of 4 points per wavelength. This result is consistent with our earlier statement that 4 points per wavelength is the lowest density threshold required to sufficient resolve the acoustic wave phenomena. 


\begin{tabular}{|l||c|c|c|c|c|}
\hline$\lambda=1$ & EBI-0 & EBI-1 & EBI-2 & EBI-3 & EBI-4 \\
\hline CGIU2 & 4091 & 3849 & 3605 & 3420 & 3401 \\
\hline CGIU3 & 1687 & 1619 & 1541 & 1525 & 1507 \\
\hline CGIU4 & 1250 & 1227 & 1223 & 1212 & 1213 \\
\hline CGIU5 & 1274 & 1277 & 1278 & 1280 & 1293 \\
\hline
\end{tabular}

Table III. Number of iterations required for the iterative methods to achieve convergence (i.e., to reduce the relative $L_{2}$ norm of the residual $\|b-A x\|_{2} /\|b\|_{2}$ below $5.10^{-7}$ ) for $k=2 \pi$. The computations are performed on a grid comprising $257^{3}$ points.

It should be noted that while in Tables I, III and II, we focus on the number of iterations required to achieve convergence, the per-iteration complexity to some extent depends on the specific method and problem size. For example, adding one more level of EBI with the configuration comprising 257 points in each direction, raises the complexity of each iteration by $3 \%$ to $9 \%$, with the exact figure depending on the number of levels of the conventional IU.

\section{CONCLUDING REMARKS}

This paper introduces a new multilevel method for an efficient solution of the acoustic scattering problem in 3D. Towards development of this technique, a new type of IU that we called Helmholtz Equation-Based Interpolation (EBI) [2] has been extended to three spatial dimensions. The proposed approach emerges from combining the efficiency of the classical IU preconditioner for the Laplace and Helmholtz equations with the ideas of the boundary element methods. Our results demonstrate an improvement in the numerical efficacy compared to the method using only the classical IU.

\section{ACKNOWLEDGEMENT}

Computational tests have been performed using Orca, the server of the Centre Commun de Calcul Intensif of the Université des Antilles et de la Guyane and Verdi, the server of the School of Electrical Engineering of Tel Aviv University.

\section{REFERENCES}

1. P. Poullet and A. Boag, Incremental unknowns preconditioning for solving the Helmholtz equation, Numer. Meth. for PDEs, 23-6 (2007) 1396-1410.

2. P. Poullet and A. Boag, Equation-based interpolation and incremental unknowns for solving the Helmholtz equation, Appl. Num. Math. 60 (2010) 1148-1156.

3. F. Ihlenburg, Finite element analysis of acoustic scattering, Applied Mathematical Sciences 132, Springer-Verlag, 1998.

4. Y.A. Erlangga, Advances in iterative methods and preconditioners for the Helmholtz equation, Arch. Comput. Meth. Eng., 15 (2008) 37-66.

5. Y.A. Erlangga, C. Vuik and C.W. Oosterlee, On a class of preconditioners for solving the Helmholtz equation, Appl. Num. Math. 50 (3-4) (2004) 409-425.

6. Y.A. Erlangga, C.W. Oosterlee and C. Vuik, A novel multigrid based preconditioner for heterogeneous Helmholtz problems, SIAM J. Sci. Comput. 27 (4) (2006) 1471-1492.

7. Y.A. Erlangga, C.W. Oosterlee and C. Vuik, Comparison of multigrid and incomplete LU shifted-Laplace preconditioners for the inhomogeneous Helmholtz equation, Appl. Num. Math. 56 (2006) 648-666.

8. H.C. Elman, O.G. Ernst and D.P. O'Leary, A multigrid method enhanced by Krylov subspace iteration for discrete Helmholtz equations, SIAM J. Sci. Comput. 23 (4) (2001) 1291-1315.

9. I. Livshits, An algebraic multigrid wave-ray algorithm to solve eignevalue problems for the Helmholtz operator, Numer. Linear Algebra Appl. 11 (2004) 229-239.

10. I. Livshits and A. Brandt, Accuracy properties of the wave-ray multigrid algorithm for Helmholtz equations, SIAM J. Sci. Comput. 28 (4) (2006) 1228-1251.

11. A. Bayliss, C.I. Goldstein and E. Turkel, The numerical solution of the Helmholtz equation for wave propagation problems in underwater acoustics, Comput. Math. Appl. 11 (1985) 655-665.

12. R. Temam, Inertial manifolds and multigrid methods, SIAM J. Math. Anal. 21 (1) (1990) 154-178.

13. H. Yserentant, On the multi-level splitting of fnite element spaces, Numer. Math. 49 (1986) 379-412. 
14. M. Chen and R. Temam, Incremental unknowns for solving partial differential equations, Num. Math. 59 (1991) $255-271$.

15. M. Chen and R. Temam, Incremental unknowns in finite differences: condition number of the matrix, SIAM J. Matrix Anal. Appl. 14 (2) (1993) 432-455.

16. S. Garcia, The matricial framework for the incremental unknowns method, Numer. Funct. Anal. Optimization 14 (1-2) (1993) 25-44.

17. M. Chen, A. Miranville and R. Temam, Incremental unknowns in finite differences in three space dimensions, Comput. Appl. Math. 14 (3) (1995) 219-252.

18. S. Garcia, Incremental Unknowns and graph techniques in three space dimensions, Appl. Num. Math. 44 (3) (2003) 329-365.

19. L. Song and Y. Wu, Incremental Unknowns in three-dimensional stationary problem, Numer. Algor. 46 (2007) $153-171$.

20. J.-P. Chehab, Incremental unknowns method and compact schemes, Math. Mod. and Numer. Anal., 32 (1) (1998) $51-83$.

21. J.-P. Chehab and A. Miranville, Incremental unknowns on nonuniform meshes, Math. Mod. and Numer. Anal., 32 (5) (1998) 539-577.

22. P. Poullet, Staggered incremental unknowns for solving Stokes and generalized Stokes problems, Appl. Num. Math., 35 (1) (2000) 23-41.

23. H. Yserentant, On the multi-level splitting of finite element spaces for indefinite elliptic boundary value problems, SIAM J. Numer. Anal. 23 (3) (1986) 581-595.

24. S. Andouze, O. Goubet and P. Poullet, A multilevel method for solving the Helmholtz equation : the analysis of the one-dimensional case. To Appear in Int. J. Numer. Anal. Model.

25. E. Heikkola, T. Rossi and J. Toivanen, A parallel fictitious domain method for the three-dimensional Helmholtz equation, SIAM J. Sci. Comput. 24-5 (2003) 1567-1588.

26. G. Mur, Absorbing boundary conditions for the finite-difference approximation of the time-domain electromagnetic-field equations, IEEE Trans. Electromagn. Compat., EMC-23 (4) (1981) 377-382.

27. A. Bamberger, P. Joly and J.E. Roberts, Second-order absorbing boundary conditions for the wave equation: a solution for the corner problem, SIAM J. Numer. Anal. 27-2 (1990) 323-352.

28. Y. Saad, Iterative Methods for Sparse Linear Systems. PWS Pulishing, New-York, 1996.

29. A. Bayliss, C.I. Goldstein and E. Turkel, An iterative method for the Helmholtz equation, J. Comput. Phys. 49, pp. 443-457, 1983. 